\title{
Donor-Stabilized Silyl Cations. 8. Carbon-Carbon Bond Formation through a Novel Interchelate Molecular Rearrangement in Pentacoordinate Siliconium-Ion Salts *
}

\author{
by \\ Boris Gostevskii, Vadim Pestunovich, Inna Kalikhman, ${ }^{*}$ Akella \\ Sivaramakrishna, Nikolaus Kocher, Stephan Deuerlein, Dirk Leusser, \\ Dietmar Stalke and Daniel Kost*
}

Department of Chemistry, Ben-Gurion University, Beer-Sheva 84105, Israel. Fax: (+972)8-647-2943. E-mail: kostd@ bgumail.bgu.ac.il (DK), Innakal@ bgumail.bgu.ac.il (IK)

\begin{abstract}
A. E. Favorsky Irkutsk Institute of Chemistry, Russian Academy of Sciences, Irkutsk, Russia.
\end{abstract}

Institut für Anorganische Chemie der Universität Würzburg, am Hubland, Würzburg, Germany.

\section{Contents:}

Data tables and figures for crystal structures of $\mathbf{3} \mathbf{a}$ and $\mathbf{3 b}$. 
$3 a$

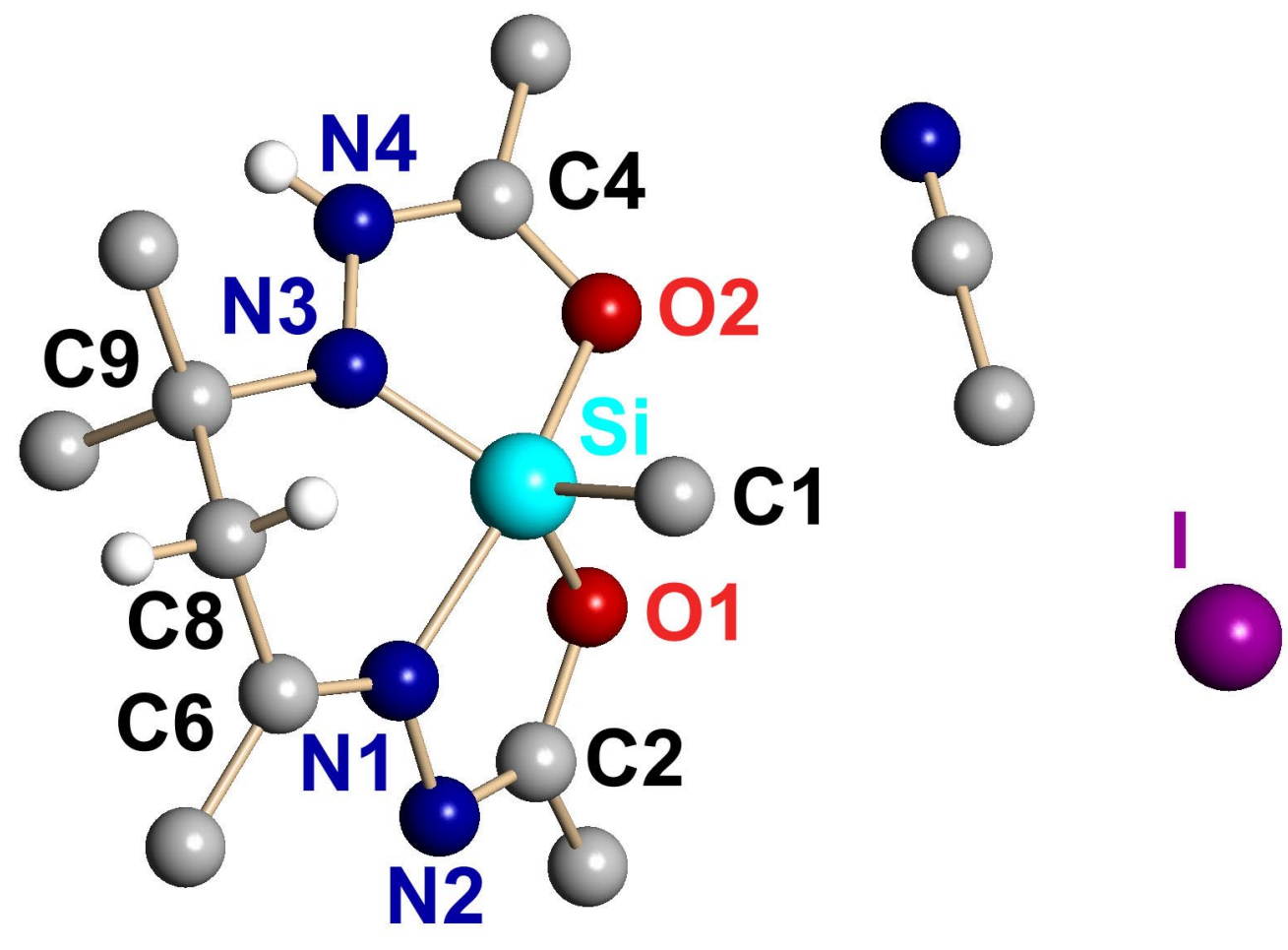

Asymmetric unit of 3a. Hydrogen atoms, except for H4, H8A, H8B are omitted for clarity. 
Table 1. Crystal data and structure refinement for 3a.

Identification code

Empirical formula

Formula weight

Temperature

Wavelength

Crystal system

Space group

Unit cell dimensions

Volume

Z

Density (calculated)

Absorption coefficient

$\mathrm{F}(000)$

Crystal size

Theta range for data collection

Index ranges

Reflections collected

Independent reflections

Completeness to theta $=26.45^{\circ}$

Refinement method

Data / restraints / parameters

Goodness-of-fit on $\mathrm{F}^{2}$

Final $R$ indices [l>2sigma(I)]

$R$ indices (all data)

Largest diff. peak and hole 3a

C13 H24 I N5 O2 Si

437.36

100(2) K

$0.71073 \AA$

Triclinic

P-1

$\mathrm{a}=8.832(2) \AA \quad \alpha=98.806(3)^{\circ}$.

$\mathrm{b}=9.853(2) \AA \quad \beta=93.522(3)^{\circ}$.

$\mathrm{c}=10.885(2) \AA \quad \gamma=101.601(3)^{\circ}$.

912.9(4) $\AA^{3}$

2

$1.591 \mathrm{Mg} / \mathrm{m}^{3}$

$1.833 \mathrm{~mm}^{-1}$

440

$0.4 \times 0.4 \times 0.4 \mathrm{~mm}^{3}$

2.14 to $26.45^{\circ}$.

$-11<=\mathrm{h}<=10,-12<=\mathrm{k}<=12,0<=\mathrm{k}<=13$

18873

$3724[\mathrm{R}$ (int) $=0.0218]$

$99.3 \%$

Full-matrix least-squares on $\mathrm{F}^{2}$

3724 / 0 / 210

1.078

$\mathrm{R} 1=0.0164, \mathrm{wR} 2=0.0432$

$\mathrm{R} 1=0.0166, \mathrm{wR} 2=0.0434$

0.541 and -0.294 e. $\AA^{-3}$ 
S4

Table 2. Atomic coordinates $\left(\mathrm{x} 10^{4}\right)$ and equivalent isotropic displacement parameters $\left(\mathrm{pm}^{2} \times 10^{-1}\right)$ for 3a. $U(e q)$ is defined as one third of the trace of the orthogonalized $U^{i j}$ tensor.

\begin{tabular}{|c|c|c|c|c|}
\hline & $x$ & $y$ & $z$ & $\mathrm{U}(\mathrm{eq})$ \\
\hline $\operatorname{Si}(1)$ & $6287(1)$ & $7076(1)$ & $3042(1)$ & $15(1)$ \\
\hline I(1) & $2670(1)$ & $7628(1)$ & $9336(1)$ & $20(1)$ \\
\hline$N(1)$ & $8254(2)$ & $6492(1)$ & $3098(1)$ & $16(1)$ \\
\hline$N(2)$ & $8849(2)$ & $6459(1)$ & $4323(1)$ & $18(1)$ \\
\hline$N(3)$ & 6898(2) & $8073(1)$ & 1901(1) & $15(1)$ \\
\hline$N(4)$ & $6148(2)$ & $9187(2)$ & 1891(1) & $16(1)$ \\
\hline $\mathrm{O}(1)$ & $6704(1)$ & $7396(1)$ & $4631(1)$ & $18(1)$ \\
\hline $\mathrm{O}(2)$ & $4857(1)$ & $8119(1)$ & $3239(1)$ & $18(1)$ \\
\hline$C(1)$ & 4999(2) & $5321(2)$ & $2555(2)$ & $25(1)$ \\
\hline $\mathrm{C}(2)$ & $7965(2)$ & $7006(2)$ & $5062(2)$ & $17(1)$ \\
\hline $\mathrm{C}(3)$ & 8353(2) & 7303(2) & $6430(2)$ & $22(1)$ \\
\hline$C(4)$ & $5055(2)$ & 9166(2) & $2627(2)$ & $17(1)$ \\
\hline$C(5)$ & $4086(2)$ & 10228(2) & $2775(2)$ & $20(1)$ \\
\hline$C(6)$ & 9099(2) & 6281(2) & 2199(2) & $16(1)$ \\
\hline $\mathrm{C}(7)$ & 10628(2) & $5888(2)$ & $2341(2)$ & $23(1)$ \\
\hline$C(8)$ & $8502(2)$ & $6555(2)$ & $970(2)$ & $17(1)$ \\
\hline $\mathrm{C}(9)$ & $8135(2)$ & $8026(2)$ & $1058(1)$ & $15(1)$ \\
\hline$C(10)$ & $9568(2)$ & $9178(2)$ & 1565(2) & $18(1)$ \\
\hline$C(11)$ & $7554(2)$ & $8179(2)$ & $-254(2)$ & $19(1)$ \\
\hline$C(20)$ & $2212(2)$ & $8416(2)$ & $5426(2)$ & $33(1)$ \\
\hline$C(21)$ & $2637(2)$ & $7108(2)$ & 5628(2) & $31(1)$ \\
\hline $\mathrm{N}(20)$ & $1890(2)$ & $9413(2)$ & $5284(2)$ & $46(1)$ \\
\hline
\end{tabular}


Table 3. Bond lengths $[\mathrm{pm}]$ and angles $\left[{ }^{\circ}\right]$ for $\mathbf{3 a}$.

\begin{tabular}{|c|c|c|c|}
\hline $\mathrm{Si}(1)-\mathrm{O}(1)$ & $1.7134(13)$ & $\mathrm{C}(10)-\mathrm{H}(10 \mathrm{C})$ & 0.9600 \\
\hline $\mathrm{Si}(1)-\mathrm{N}(3)$ & $1.7434(14)$ & $C(11)-H(11 A)$ & 0.9600 \\
\hline $\mathrm{Si}(1)-\mathrm{O}(2)$ & $1.7843(12)$ & $\mathrm{C}(11)-\mathrm{H}(11 \mathrm{~B})$ & 0.9600 \\
\hline $\mathrm{Si}(1)-C(1)$ & $1.8492(18)$ & $\mathrm{C}(11)-\mathrm{H}(11 \mathrm{C})$ & 0.9600 \\
\hline $\mathrm{Si}(1)-N(1)$ & $1.9372(14)$ & $\mathrm{C}(20)-\mathrm{N}(20)$ & $1.106(3)$ \\
\hline$N(1)-C(6)$ & $1.283(2)$ & $C(20)-C(21)$ & $1.456(3)$ \\
\hline$N(1)-N(2)$ & $1.4105(19)$ & $\mathrm{C}(21)-\mathrm{H}(21 \mathrm{~A})$ & 0.9600 \\
\hline$N(2)-C(2)$ & $1.289(2)$ & $\mathrm{C}(21)-\mathrm{H}(21 \mathrm{~B})$ & 0.9600 \\
\hline$N(3)-N(4)$ & $1.3929(19)$ & $\mathrm{C}(21)-\mathrm{H}(21 \mathrm{C})$ & 0.9600 \\
\hline$N(3)-C(9)$ & $1.4724(19)$ & $\mathrm{O}(1)-\mathrm{Si}(1)-\mathrm{N}(3)$ & $131.41(7)$ \\
\hline$N(4)-C(4)$ & $1.290(2)$ & $\mathrm{O}(1)-\mathrm{Si}(1)-\mathrm{O}(2)$ & $88.50(6)$ \\
\hline $\mathrm{N}(4)-\mathrm{H}(4)$ & $0.83(2)$ & $\mathrm{N}(3)-\mathrm{Si}(1)-\mathrm{O}(2)$ & $85.24(6)$ \\
\hline $\mathrm{O}(1)-\mathrm{C}(2)$ & $1.330(2)$ & $O(1)-S i(1)-C(1)$ & 109.64(7) \\
\hline $\mathrm{O}(2)-\mathrm{C}(4)$ & $1.300(2)$ & $N(3)-S i(1)-C(1)$ & $118.92(8)$ \\
\hline$C(1)-H(1 A)$ & 0.9600 & $\mathrm{O}(2)-\mathrm{Si}(1)-\mathrm{C}(1)$ & $99.48(7)$ \\
\hline$C(1)-H(1 B)$ & 0.9600 & $\mathrm{O}(1)-\mathrm{Si}(1)-\mathrm{N}(1)$ & $82.03(6)$ \\
\hline$C(1)-H(1 C)$ & 0.9600 & $N(3)-S i(1)-N(1)$ & $89.52(6)$ \\
\hline$C(2)-C(3)$ & $1.479(2)$ & $\mathrm{O}(2)-\mathrm{Si}(1)-\mathrm{N}(1)$ & $161.93(6)$ \\
\hline $\mathrm{C}(3)-\mathrm{H}(3 \mathrm{~A})$ & 0.9600 & $C(1)-S i(1)-N(1)$ & $98.19(7)$ \\
\hline $\mathrm{C}(3)-\mathrm{H}(3 \mathrm{~B})$ & 0.9600 & $C(6)-N(1)-N(2)$ & $118.73(13)$ \\
\hline $\mathrm{C}(3)-\mathrm{H}(3 \mathrm{C})$ & 0.9600 & $C(6)-N(1)-S i(1)$ & $128.06(12)$ \\
\hline$C(4)-C(5)$ & $1.476(2)$ & $N(2)-N(1)-S i(1)$ & $112.82(10)$ \\
\hline $\mathrm{C}(5)-\mathrm{H}(5 \mathrm{~A})$ & 0.9600 & $C(2)-N(2)-N(1)$ & $106.41(13)$ \\
\hline $\mathrm{C}(5)-\mathrm{H}(5 \mathrm{~B})$ & 0.9600 & $N(4)-N(3)-C(9)$ & $115.82(12)$ \\
\hline $\mathrm{C}(5)-\mathrm{H}(5 \mathrm{C})$ & 0.9600 & $N(4)-N(3)-S i(1)$ & $111.90(10)$ \\
\hline $\mathrm{C}(6)-\mathrm{C}(7)$ & $1.483(2)$ & $C(9)-N(3)-S i(1)$ & $131.98(11)$ \\
\hline$C(6)-C(8)$ & $1.491(2)$ & $\mathrm{C}(4)-\mathrm{N}(4)-\mathrm{N}(3)$ & $113.46(14)$ \\
\hline $\mathrm{C}(7)-\mathrm{H}(7 \mathrm{~A})$ & 0.9600 & $\mathrm{C}(4)-\mathrm{N}(4)-\mathrm{H}(4)$ & $123.3(15)$ \\
\hline $\mathrm{C}(7)-\mathrm{H}(7 \mathrm{~B})$ & 0.9600 & $\mathrm{~N}(3)-\mathrm{N}(4)-\mathrm{H}(4)$ & 122.6(16) \\
\hline $\mathrm{C}(7)-\mathrm{H}(7 \mathrm{C})$ & 0.9600 & $\mathrm{C}(2)-\mathrm{O}(1)-\mathrm{Si}(1)$ & $116.25(10)$ \\
\hline $\mathrm{C}(8)-\mathrm{C}(9)$ & $1.538(2)$ & $\mathrm{C}(4)-\mathrm{O}(2)-\mathrm{Si}(1)$ & $113.87(10)$ \\
\hline $\mathrm{C}(8)-\mathrm{H}(8 \mathrm{~A})$ & 0.9700 & $\mathrm{Si}(1)-\mathrm{C}(1)-\mathrm{H}(1 \mathrm{~A})$ & 109.5 \\
\hline $\mathrm{C}(8)-\mathrm{H}(8 \mathrm{~B})$ & 0.9700 & $\mathrm{Si}(1)-\mathrm{C}(1)-\mathrm{H}(1 \mathrm{~B})$ & 109.5 \\
\hline$C(9)-C(11)$ & $1.528(2)$ & $H(1 A)-C(1)-H(1 B)$ & 109.5 \\
\hline$C(9)-C(10)$ & $1.530(2)$ & $\mathrm{Si}(1)-\mathrm{C}(1)-\mathrm{H}(1 \mathrm{C})$ & 109.5 \\
\hline$C(10)-H(10 A)$ & 0.9600 & $H(1 A)-C(1)-H(1 C)$ & 109.5 \\
\hline $\mathrm{C}(10)-\mathrm{H}(10 \mathrm{~B})$ & 0.9600 & $H(1 B)-C(1)-H(1 C)$ & 109.5 \\
\hline
\end{tabular}




\begin{tabular}{|c|c|c|c|}
\hline$N(2)-C(2)-O(1)$ & $121.85(15)$ & $C(10)-C(9)-C(8)$ & $111.38(13)$ \\
\hline$N(2)-C(2)-C(3)$ & $121.38(15)$ & $C(9)-C(10)-H(10 A)$ & 109.5 \\
\hline $\mathrm{O}(1)-\mathrm{C}(2)-\mathrm{C}(3)$ & $116.69(14)$ & $\mathrm{C}(9)-\mathrm{C}(10)-\mathrm{H}(10 \mathrm{~B})$ & 109.5 \\
\hline $\mathrm{C}(2)-\mathrm{C}(3)-\mathrm{H}(3 \mathrm{~A})$ & 109.5 & $H(10 A)-C(10)-H(10 B)$ & 109.5 \\
\hline $\mathrm{C}(2)-\mathrm{C}(3)-\mathrm{H}(3 \mathrm{~B})$ & 109.5 & $C(9)-C(10)-H(10 C)$ & 109.5 \\
\hline$H(3 A)-C(3)-H(3 B)$ & 109.5 & $H(10 A)-C(10)-H(10 C)$ & 109.5 \\
\hline $\mathrm{C}(2)-\mathrm{C}(3)-\mathrm{H}(3 \mathrm{C})$ & 109.5 & $H(10 B)-C(10)-H(10 C)$ & 109.5 \\
\hline$H(3 A)-C(3)-H(3 C)$ & 109.5 & $C(9)-C(11)-H(11 A)$ & 109.5 \\
\hline$H(3 B)-C(3)-H(3 C)$ & 109.5 & $\mathrm{C}(9)-\mathrm{C}(11)-\mathrm{H}(11 \mathrm{~B})$ & 109.5 \\
\hline $\mathrm{N}(4)-\mathrm{C}(4)-\mathrm{O}(2)$ & $114.63(14)$ & $H(11 A)-C(11)-H(11 B)$ & 109.5 \\
\hline$N(4)-C(4)-C(5)$ & $123.69(15)$ & $C(9)-C(11)-H(11 C)$ & 109.5 \\
\hline $\mathrm{O}(2)-\mathrm{C}(4)-\mathrm{C}(5)$ & $121.68(14)$ & $H(11 A)-C(11)-H(11 C)$ & 109.5 \\
\hline $\mathrm{C}(4)-\mathrm{C}(5)-\mathrm{H}(5 \mathrm{~A})$ & 109.5 & $H(11 B)-C(11)-H(11 C)$ & 109.5 \\
\hline $\mathrm{C}(4)-\mathrm{C}(5)-\mathrm{H}(5 \mathrm{~B})$ & 109.5 & $N(20)-C(20)-C(21)$ & $179.4(2)$ \\
\hline$H(5 A)-C(5)-H(5 B)$ & 109.5 & $C(20)-C(21)-H(21 A)$ & 109.5 \\
\hline $\mathrm{C}(4)-\mathrm{C}(5)-\mathrm{H}(5 \mathrm{C})$ & 109.5 & $C(20)-C(21)-H(21 B)$ & 109.5 \\
\hline$H(5 A)-C(5)-H(5 C)$ & 109.5 & $H(21 A)-C(21)-H(21 B)$ & 109.5 \\
\hline$H(5 B)-C(5)-H(5 C)$ & 109.5 & $C(20)-C(21)-H(21 C)$ & 109.5 \\
\hline$N(1)-C(6)-C(7)$ & $124.32(15)$ & $H(21 A)-C(21)-H(21 C)$ & 109.5 \\
\hline$N(1)-C(6)-C(8)$ & $115.53(14)$ & $H(21 B)-C(21)-H(21 C)$ & 109.5 \\
\hline$C(7)-C(6)-C(8)$ & $120.01(14)$ & & \\
\hline $\mathrm{C}(6)-\mathrm{C}(7)-\mathrm{H}(7 \mathrm{~A})$ & 109.5 & & \\
\hline $\mathrm{C}(6)-\mathrm{C}(7)-\mathrm{H}(7 \mathrm{~B})$ & 109.5 & & \\
\hline$H(7 A)-C(7)-H(7 B)$ & 109.5 & & \\
\hline $\mathrm{C}(6)-\mathrm{C}(7)-\mathrm{H}(7 \mathrm{C})$ & 109.5 & & \\
\hline$H(7 A)-C(7)-H(7 C)$ & 109.5 & & \\
\hline $\mathrm{H}(7 \mathrm{~B})-\mathrm{C}(7)-\mathrm{H}(7 \mathrm{C})$ & 109.5 & & \\
\hline$C(6)-C(8)-C(9)$ & $112.43(13)$ & & \\
\hline $\mathrm{C}(6)-\mathrm{C}(8)-\mathrm{H}(8 \mathrm{~A})$ & 109.1 & & \\
\hline $\mathrm{C}(9)-\mathrm{C}(8)-\mathrm{H}(8 \mathrm{~A})$ & 109.1 & & \\
\hline $\mathrm{C}(6)-\mathrm{C}(8)-\mathrm{H}(8 \mathrm{~B})$ & 109.1 & & \\
\hline $\mathrm{C}(9)-\mathrm{C}(8)-\mathrm{H}(8 \mathrm{~B})$ & 109.1 & & \\
\hline$H(8 A)-C(8)-H(8 B)$ & 107.8 & & \\
\hline$N(3)-C(9)-C(11)$ & $110.40(13)$ & & \\
\hline$N(3)-C(9)-C(10)$ & $110.39(13)$ & & \\
\hline$C(11)-C(9)-C(10)$ & $110.65(13)$ & & \\
\hline$N(3)-C(9)-C(8)$ & $106.67(12)$ & & \\
\hline$C(11)-C(9)-C(8)$ & $107.23(13)$ & & \\
\hline
\end{tabular}


Table 4. Anisotropic displacement parameters $\left(\mathrm{pm}^{2} \times 10^{-1}\right)$ for $\mathbf{3 a}$. The anisotropic displacement factor exponent takes the form: $-2 \pi^{2}\left[h^{2} a^{* 2} U^{11}+\ldots+2 h k a^{*} b^{*} U^{12}\right]$

\begin{tabular}{|c|c|c|c|c|c|c|}
\hline & $U^{11}$ & $U^{22}$ & $u^{33}$ & $u^{23}$ & $U^{13}$ & $U^{12}$ \\
\hline $\mathrm{Si}(1)$ & $13(1)$ & $14(1)$ & $17(1)$ & $3(1)$ & $4(1)$ & $3(1)$ \\
\hline $\mathrm{I}(1)$ & $21(1)$ & $17(1)$ & $22(1)$ & $4(1)$ & $7(1)$ & $5(1)$ \\
\hline $\mathrm{N}(1)$ & $16(1)$ & $13(1)$ & $17(1)$ & $3(1)$ & 1(1) & $3(1)$ \\
\hline $\mathrm{N}(2)$ & $20(1)$ & $17(1)$ & $17(1)$ & $4(1)$ & $1(1)$ & $4(1)$ \\
\hline $\mathrm{N}(3)$ & $14(1)$ & $17(1)$ & $18(1)$ & $5(1)$ & $5(1)$ & $7(1)$ \\
\hline $\mathrm{N}(4)$ & $16(1)$ & $15(1)$ & $18(1)$ & $4(1)$ & $3(1)$ & $5(1)$ \\
\hline $\mathrm{O}(1)$ & $20(1)$ & $19(1)$ & $17(1)$ & $4(1)$ & $5(1)$ & $6(1)$ \\
\hline $\mathrm{O}(2)$ & $16(1)$ & $19(1)$ & $22(1)$ & $6(1)$ & $6(1)$ & $6(1)$ \\
\hline$C(1)$ & $20(1)$ & $19(1)$ & $33(1)$ & $2(1)$ & $5(1)$ & $1(1)$ \\
\hline$C(2)$ & $18(1)$ & $13(1)$ & $20(1)$ & $5(1)$ & $3(1)$ & $1(1)$ \\
\hline$C(3)$ & $25(1)$ & $22(1)$ & $18(1)$ & $4(1)$ & $3(1)$ & $3(1)$ \\
\hline$C(4)$ & $14(1)$ & $18(1)$ & $18(1)$ & $2(1)$ & $1(1)$ & $3(1)$ \\
\hline$C(5)$ & $18(1)$ & $22(1)$ & $23(1)$ & $4(1)$ & $4(1)$ & $9(1)$ \\
\hline$C(6)$ & $17(1)$ & $12(1)$ & $20(1)$ & $0(1)$ & $3(1)$ & $3(1)$ \\
\hline$C(7)$ & $20(1)$ & $25(1)$ & $25(1)$ & $4(1)$ & $5(1)$ & 11(1) \\
\hline$C(8)$ & $18(1)$ & $17(1)$ & $17(1)$ & 1(1) & $5(1)$ & $5(1)$ \\
\hline $\mathrm{C}(9)$ & $14(1)$ & $16(1)$ & $15(1)$ & $2(1)$ & $4(1)$ & $3(1)$ \\
\hline$C(10)$ & $14(1)$ & $18(1)$ & $22(1)$ & $2(1)$ & $3(1)$ & $3(1)$ \\
\hline$C(11)$ & $21(1)$ & $21(1)$ & $16(1)$ & $2(1)$ & $3(1)$ & $5(1)$ \\
\hline$C(20)$ & $28(1)$ & $34(1)$ & $29(1)$ & $-2(1)$ & $6(1)$ & $-8(1)$ \\
\hline$C(21)$ & $29(1)$ & $36(1)$ & $24(1)$ & $5(1)$ & $2(1)$ & $2(1)$ \\
\hline $\mathrm{N}(20)$ & $38(1)$ & $37(1)$ & $61(1)$ & $10(1)$ & $9(1)$ & $-2(1)$ \\
\hline
\end{tabular}




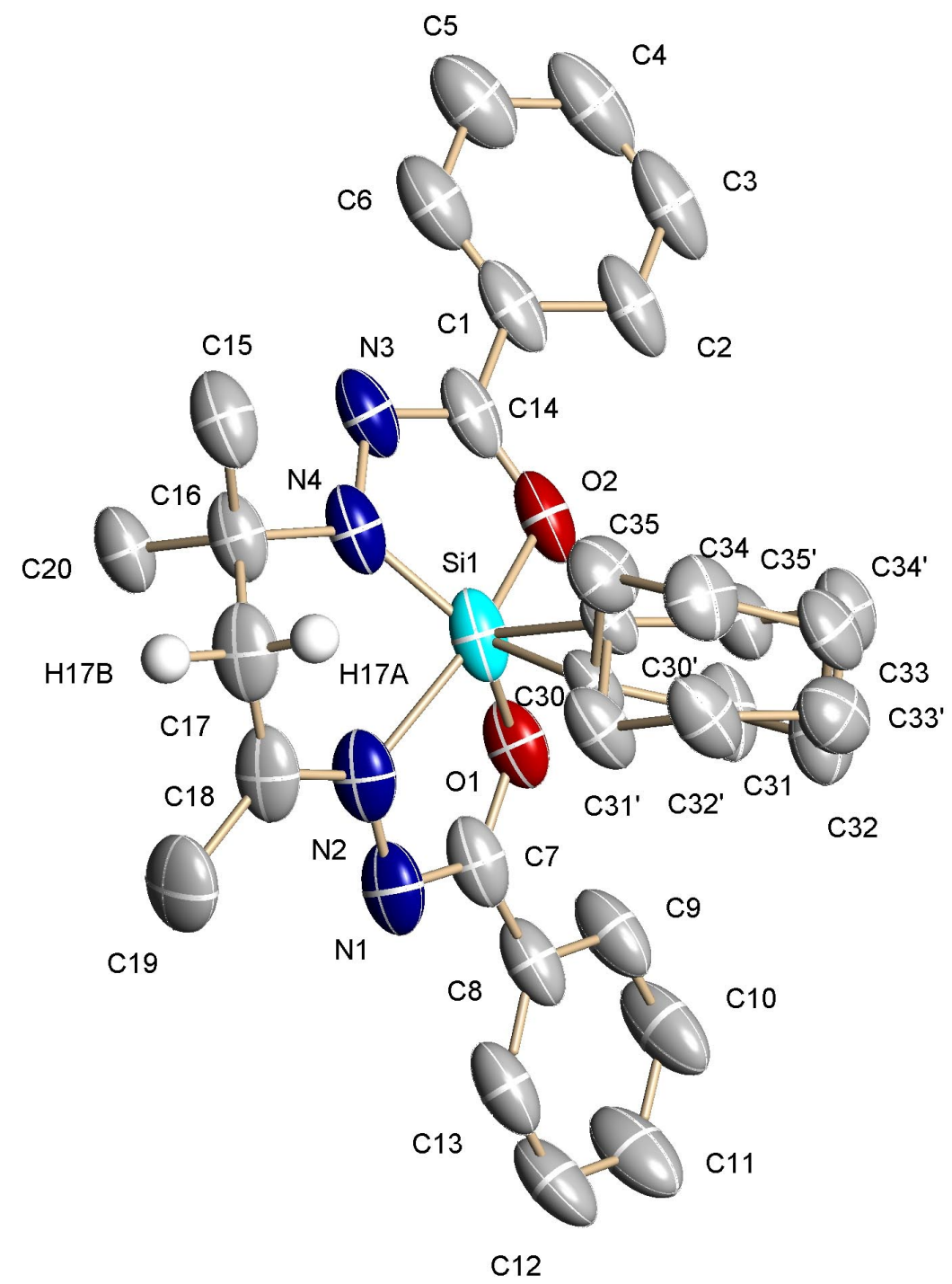

Asymmetric unit of $\mathbf{3 b}$. Hydrogen atoms, except H17A and H17B, are omitted for clarity. The anisotropic displacement parameters are depicted at the $50 \%$ probability level. 
Table 1. Crystal data and structure refinement for $\mathbf{3 b}$.

Identification code

Empirical formula

Formula weight

Temperature

Wavelength

Crystal system

Space group

Unit cell dimensions

Volume

$\mathrm{Z}$

Density (calculated)

Absorption coefficient

$\mathrm{F}(000)$

Crystal size

Theta range for data collection

Index ranges

Reflections collected

Independent reflections

Completeness to theta $=26.49^{\circ}$

Absorption correction

Max. and min. transmission

Refinement method

Data / restraints / parameters

Goodness-of-fit on $\mathrm{F}^{2}$

Final $\mathrm{R}$ indices [I $>2 \operatorname{sigma}(\mathrm{I})]$

$\mathrm{R}$ indices (all data)

Absolute structure parameter

Largest diff. peak and hole gb341

C26 H32 N4 O2 Si

460.64

100(2) K

$71.073 \mathrm{pm}$

Orthorombic

Pca2(1)

$\mathrm{a}=2334.5(4) \mathrm{pm} \quad \alpha=90^{\circ}$.

$\mathrm{b}=965.61(17) \mathrm{pm} \quad \beta=90^{\circ}$.

$\mathrm{c}=1079.69(19) \mathrm{pm} \quad \gamma=90^{\circ}$.

$2.4338(7) \mathrm{nm}^{3}$

8

$1.257 \mathrm{Mg} / \mathrm{m}^{3}$

$0.127 \mathrm{~mm}^{-1}$

984

$0.4 \times 0.4 \times 0.3 \mathrm{~mm}^{3}$

2.57 to $26.49^{\circ}$.

$0<=\mathrm{h}<=29,0<=\mathrm{k}<=12,-13<=\mathrm{l}<=13$

32267

$5003[\mathrm{R}(\mathrm{int})=0.0279]$

$99.4 \%$

Empirical

0.84 and 0.98

Full-matrix least-squares on $\mathrm{F}^{2}$

$5003 / 14 / 357$

1.077

$\mathrm{R} 1=0.0357, \mathrm{wR} 2=0.0904$

$\mathrm{R} 1=0.0421, \mathrm{wR} 2=0.0956$

$0.06(11)$

0.244 and -0.253 e. $\AA^{-3}$ 
S10

Table 2. Atomic coordinates ( $\left.\times 10^{4}\right)$ and equivalent isotropic displacement parameters $\left(\mathrm{pm}^{2} \times 10^{-1}\right)$ for $\mathbf{3 b}$. $U(e q)$ is defined as one third of the trace of the orthogonalized $U^{i j}$ tensor.

\begin{tabular}{|c|c|c|c|c|}
\hline & $\mathrm{x}$ & $\mathrm{y}$ & $\mathrm{z}$ & $\mathrm{U}(\mathrm{eq})$ \\
\hline $\mathrm{C}(1)$ & 10021(1) & $3230(2)$ & $6625(2)$ & $64(1)$ \\
\hline $\mathrm{C}(2)$ & $9727(1)$ & $3557(2)$ & $5534(2)$ & $73(1)$ \\
\hline $\mathrm{C}(3)$ & $10021(2)$ & 4093(2) & $4523(2)$ & $85(1)$ \\
\hline $\mathrm{C}(4)$ & $10599(2)$ & $4299(2)$ & $4577(2)$ & $88(1)$ \\
\hline$C(5)$ & 10899(1) & $3959(2)$ & $5640(2)$ & $83(1)$ \\
\hline$C(6)$ & $10610(1)$ & $3431(2)$ & $6663(2)$ & $74(1)$ \\
\hline $\mathrm{C}(7)$ & 7997(1) & $3421(2)$ & $9979(2)$ & $59(1)$ \\
\hline $\mathrm{C}(8)$ & $7483(1)$ & $4288(2)$ & $9871(2)$ & $60(1)$ \\
\hline $\mathrm{C}(9)$ & $7397(1)$ & $5117(2)$ & $8831(2)$ & $70(1)$ \\
\hline$C(10)$ & 6911(1) & $5917(3)$ & $8729(2)$ & $87(1)$ \\
\hline $\mathrm{C}(11)$ & $6510(1)$ & $5921(3)$ & $9666(2)$ & $90(1)$ \\
\hline $\mathrm{C}(12)$ & $6593(1)$ & $5102(2)$ & $10710(2)$ & $80(1)$ \\
\hline $\mathrm{C}(13)$ & 7071(1) & $4284(2)$ & 10802(2) & $68(1)$ \\
\hline$C(14)$ & $9714(1)$ & $2680(2)$ & $7700(2)$ & $59(1)$ \\
\hline$C(15)$ & 10294(1) & $277(2)$ & $10398(2)$ & $71(1)$ \\
\hline$C(16)$ & 9819(1) & $1307(2)$ & $10697(2)$ & $57(1)$ \\
\hline$C(17)$ & $9356(1)$ & $548(2)$ & $11454(2)$ & $68(1)$ \\
\hline $\mathrm{C}(18)$ & $8844(1)$ & $1406(2)$ & $11740(2)$ & $66(1)$ \\
\hline $\mathrm{C}(19)$ & $8639(1)$ & $1584(3)$ & $13040(2)$ & $82(1)$ \\
\hline$C(20)$ & $10055(1)$ & $2540(2)$ & $11434(2)$ & $60(1)$ \\
\hline $\mathrm{N}(1)$ & $8103(1)$ & $2762(2)$ & 10998(2) & $64(1)$ \\
\hline $\mathrm{N}(2)$ & $8598(1)$ & $1980(2)$ & $10800(2)$ & $62(1)$ \\
\hline $\mathrm{N}(3)$ & $9970(1)$ & $2256(2)$ & $8683(1)$ & $60(1)$ \\
\hline $\mathrm{N}(4)$ & $9555(1)$ & $1810(2)$ & $9536(1)$ & $57(1)$ \\
\hline $\mathrm{O}(1)$ & $8348(1)$ & $3343(1)$ & 9013(1) & $59(1)$ \\
\hline $\mathrm{O}(2)$ & $9138(1)$ & $2635(1)$ & $7666(1)$ & $62(1)$ \\
\hline $\operatorname{Si}(1)$ & $8849(1)$ & 2002(1) & $9039(1)$ & $58(1)$ \\
\hline $\mathrm{C}(30)$ & $8371(2)$ & $444(4)$ & $8702(4)$ & $43(1)$ \\
\hline $\mathrm{C}(31)$ & $7936(2)$ & $759(4)$ & $7670(5)$ & $70(1)$ \\
\hline $\mathrm{C}(32)$ & $7595(2)$ & $-521(4)$ & 7303(4) & $69(1)$ \\
\hline $\mathrm{C}(33)$ & $7978(2)$ & $-1682(4)$ & 6894(4) & $62(1)$ \\
\hline$C(34)$ & $8419(2)$ & $-2032(3)$ & 7898(4) & $65(1)$ \\
\hline$C(35)$ & $8758(2)$ & $-743(4)$ & $8290(4)$ & $63(1)$ \\
\hline $\mathrm{C}\left(30^{\prime}\right)$ & $8566(2)$ & $367(4)$ & $8245(5)$ & $41(1)$ \\
\hline
\end{tabular}




\begin{tabular}{lrrrr} 
& \multicolumn{5}{c}{$\mathrm{S} 11$} \\
$\mathrm{C}\left(31^{\prime}\right)$ & $8182(2)$ & $-456(4)$ & $9110(4)$ & $61(1)$ \\
$\mathrm{C}\left(32^{\prime}\right)$ & $7961(3)$ & $-1791(5)$ & $8470(4)$ & $67(2)$ \\
$\mathrm{C}\left(33^{\prime}\right)$ & $7646(3)$ & $-1439(7)$ & $7289(6)$ & $66(1)$ \\
$\mathrm{C}\left(34^{\prime}\right)$ & $8020(2)$ & $-604(5)$ & $6410(4)$ & $62(1)$ \\
$\mathrm{C}\left(35^{\prime}\right)$ & $8241(2)$ & $708(5)$ & $7036(4)$ & $54(1)$ \\
\hline
\end{tabular}


Table 3. Bond lengths $[\mathrm{pm}]$ and angles $\left[{ }^{\circ}\right]$ for $\mathbf{3 b}$.

\begin{tabular}{|c|c|c|c|}
\hline$C(1)-C(6)$ & $138.8(4)$ & $\mathrm{C}(18)-\mathrm{N}(2)$ & $129.1(2)$ \\
\hline $\mathrm{C}(1)-\mathrm{C}(2)$ & $139.9(3)$ & $\mathrm{C}(18)-\mathrm{C}(19)$ & 149.2(3) \\
\hline $\mathrm{C}(1)-\mathrm{C}(14)$ & $146.4(3)$ & $\mathrm{C}(19)-\mathrm{H}(19 \mathrm{~A})$ & 98.00 \\
\hline $\mathrm{C}(2)-\mathrm{C}(3)$ & $139.0(4)$ & $\mathrm{C}(19)-\mathrm{H}(19 \mathrm{~B})$ & 98.00 \\
\hline $\mathrm{C}(2)-\mathrm{H}(2)$ & 95.00 & $\mathrm{C}(19)-\mathrm{H}(19 \mathrm{C})$ & 98.00 \\
\hline $\mathrm{C}(3)-\mathrm{C}(4)$ & $136.5(4)$ & $\mathrm{C}(20)-\mathrm{H}(20 \mathrm{~A})$ & 98.00 \\
\hline $\mathrm{C}(3)-\mathrm{H}(3)$ & 95.00 & $\mathrm{C}(20)-\mathrm{H}(20 \mathrm{~B})$ & 98.00 \\
\hline$C(4)-C(5)$ & $138.5(4)$ & $\mathrm{C}(20)-\mathrm{H}(20 \mathrm{C})$ & 98.00 \\
\hline $\mathrm{C}(4)-\mathrm{H}(4)$ & 95.00 & $\mathrm{~N}(1)-\mathrm{N}(2)$ & $139.8(3)$ \\
\hline$C(5)-C(6)$ & 139.1(3) & $\mathrm{N}(2)-\mathrm{Si}(1)$ & 198.95(19) \\
\hline $\mathrm{C}(5)-\mathrm{H}(5)$ & 95.00 & $\mathrm{~N}(3)-\mathrm{N}(4)$ & $140.4(2)$ \\
\hline $\mathrm{C}(6)-\mathrm{H}(6)$ & 95.00 & $\mathrm{~N}(4)-\mathrm{Si}(1)$ & $174.4(2)$ \\
\hline $\mathrm{C}(7)-\mathrm{N}(1)$ & $129.5(2)$ & $\mathrm{O}(1)-\mathrm{Si}(1)$ & $174.44(14)$ \\
\hline $\mathrm{C}(7)-\mathrm{O}(1)$ & $132.8(3)$ & $\mathrm{O}(2)-\mathrm{Si}(1)$ & $173.96(16)$ \\
\hline$C(7)-C(8)$ & $146.8(3)$ & $\mathrm{Si}(1)-\mathrm{C}(30)$ & $190.7(4)$ \\
\hline $\mathrm{C}(8)-\mathrm{C}(13)$ & $139.3(3)$ & $\mathrm{Si}(1)-\mathrm{C}\left(30^{\prime}\right)$ & $191.4(4)$ \\
\hline $\mathrm{C}(8)-\mathrm{C}(9)$ & $139.3(3)$ & $\mathrm{C}(30)-\mathrm{C}(35)$ & $152.5(5)$ \\
\hline$C(9)-C(10)$ & $137.5(3)$ & $C(30)-C(31)$ & $153.8(5)$ \\
\hline $\mathrm{C}(9)-\mathrm{H}(9)$ & 95.00 & $\mathrm{C}(30)-\mathrm{H}(30)$ & 100.00 \\
\hline$C(10)-C(11)$ & $137.9(4)$ & $C(31)-C(32)$ & $152.3(5)$ \\
\hline $\mathrm{C}(10)-\mathrm{H}(10)$ & 95.00 & $\mathrm{C}(31)-\mathrm{H}(31 \mathrm{~A})$ & 99.00 \\
\hline $\mathrm{C}(11)-\mathrm{C}(12)$ & $139.0(3)$ & $\mathrm{C}(31)-\mathrm{H}(31 \mathrm{~B})$ & 99.00 \\
\hline $\mathrm{C}(11)-\mathrm{H}(11)$ & 95.00 & $\mathrm{C}(32)-\mathrm{C}(33)$ & $150.0(6)$ \\
\hline $\mathrm{C}(12)-\mathrm{C}(13)$ & $137.0(3)$ & $\mathrm{C}(32)-\mathrm{H}(32 \mathrm{~A})$ & 99.00 \\
\hline $\mathrm{C}(12)-\mathrm{H}(12)$ & 95.00 & $\mathrm{C}(32)-\mathrm{H}(32 \mathrm{~B})$ & 99.00 \\
\hline $\mathrm{C}(13)-\mathrm{H}(13)$ & 95.00 & $\mathrm{C}(33)-\mathrm{C}(34)$ & $153.3(6)$ \\
\hline $\mathrm{C}(14)-\mathrm{N}(3)$ & $128.5(3)$ & $\mathrm{C}(33)-\mathrm{H}(33 \mathrm{~A})$ & 99.00 \\
\hline $\mathrm{C}(14)-\mathrm{O}(2)$ & $134.6(3)$ & $\mathrm{C}(33)-\mathrm{H}(33 \mathrm{~B})$ & 99.00 \\
\hline$C(15)-C(16)$ & $152.4(3)$ & $\mathrm{C}(34)-\mathrm{C}(35)$ & $153.3(5)$ \\
\hline $\mathrm{C}(15)-\mathrm{H}(15 \mathrm{~A})$ & 98.00 & $\mathrm{C}(34)-\mathrm{H}(34 \mathrm{~A})$ & 99.00 \\
\hline $\mathrm{C}(15)-\mathrm{H}(15 \mathrm{~B})$ & 98.00 & $\mathrm{C}(34)-\mathrm{H}(34 \mathrm{~B})$ & 99.00 \\
\hline $\mathrm{C}(15)-\mathrm{H}(15 \mathrm{C})$ & 98.00 & $\mathrm{C}(35)-\mathrm{H}(35 \mathrm{~A})$ & 99.00 \\
\hline $\mathrm{C}(16)-\mathrm{N}(4)$ & $147.9(2)$ & $\mathrm{C}(35)-\mathrm{H}(35 \mathrm{~B})$ & 99.00 \\
\hline $\mathrm{C}(16)-\mathrm{C}(20)$ & $153.4(3)$ & $\mathrm{C}\left(30^{\prime}\right)-\mathrm{C}\left(31^{\prime}\right)$ & $151.8(6)$ \\
\hline$C(16)-C(17)$ & $154.0(3)$ & $\mathrm{C}\left(30^{\prime}\right)-\mathrm{C}\left(35^{\prime}\right)$ & $154.5(6)$ \\
\hline$C(17)-C(18)$ & $148.7(3)$ & $\mathrm{C}\left(30^{\prime}\right)-\mathrm{H}\left(30^{\prime}\right)$ & 100.00 \\
\hline $\mathrm{C}(17)-\mathrm{H}(17 \mathrm{~A})$ & 99.00 & $\mathrm{C}\left(31^{\prime}\right)-\mathrm{C}\left(32^{\prime}\right)$ & $155.1(6)$ \\
\hline $\mathrm{C}(17)-\mathrm{H}(17 \mathrm{~B})$ & 99.00 & $\mathrm{C}\left(31^{\prime}\right)-\mathrm{H}(31 \mathrm{C})$ & 99.00 \\
\hline
\end{tabular}




\begin{tabular}{|c|c|c|c|}
\hline $\mathrm{C}\left(31^{\prime}\right)-\mathrm{H}(31 \mathrm{D})$ & 99.00 & $\mathrm{C}(8)-\mathrm{C}(9)-\mathrm{H}(9)$ & 119.8 \\
\hline $\mathrm{C}\left(32^{\prime}\right)-\mathrm{C}\left(33^{\prime}\right)$ & $151.1(7)$ & $\mathrm{C}(9)-\mathrm{C}(10)-\mathrm{C}(11)$ & $120.2(2)$ \\
\hline $\mathrm{C}\left(32^{\prime}\right)-\mathrm{H}(32 \mathrm{C})$ & 99.00 & $\mathrm{C}(9)-\mathrm{C}(10)-\mathrm{H}(10)$ & 119.9 \\
\hline $\mathrm{C}\left(32^{\prime}\right)-\mathrm{H}(32 \mathrm{D})$ & 99.00 & $\mathrm{C}(11)-\mathrm{C}(10)-\mathrm{H}(10)$ & 119.9 \\
\hline $\mathrm{C}\left(33^{\prime}\right)-\mathrm{C}\left(34^{\prime}\right)$ & $152.1(8)$ & $C(10)-C(11)-C(12)$ & $119.9(2)$ \\
\hline C(33')-H(33C) & 99.00 & $\mathrm{C}(10)-\mathrm{C}(11)-\mathrm{H}(11)$ & 120.0 \\
\hline $\mathrm{C}\left(33^{\prime}\right)-\mathrm{H}(33 \mathrm{D})$ & 99.00 & $\mathrm{C}(12)-\mathrm{C}(11)-\mathrm{H}(11)$ & 120.0 \\
\hline $\mathrm{C}\left(34^{\prime}\right)-\mathrm{C}\left(35^{\prime}\right)$ & $152.5(6)$ & $C(13)-C(12)-C(11)$ & $119.9(2)$ \\
\hline $\mathrm{C}\left(34^{\prime}\right)-\mathrm{H}(34 \mathrm{C})$ & 99.00 & $\mathrm{C}(13)-\mathrm{C}(12)-\mathrm{H}(12)$ & 120.0 \\
\hline $\mathrm{C}\left(34^{\prime}\right)-\mathrm{H}(34 \mathrm{D})$ & 99.00 & $\mathrm{C}(11)-\mathrm{C}(12)-\mathrm{H}(12)$ & 120.0 \\
\hline $\mathrm{C}\left(35^{\prime}\right)-\mathrm{H}(35 \mathrm{C})$ & 99.00 & $\mathrm{C}(12)-\mathrm{C}(13)-\mathrm{C}(8)$ & $120.67(19)$ \\
\hline $\mathrm{C}\left(35^{\prime}\right)-\mathrm{H}(35 \mathrm{D})$ & 99.00 & $\mathrm{C}(12)-\mathrm{C}(13)-\mathrm{H}(13)$ & 119.7 \\
\hline$C(6)-C(1)-C(2)$ & $118.6(2)$ & $\mathrm{C}(8)-\mathrm{C}(13)-\mathrm{H}(13)$ & 119.7 \\
\hline $\mathrm{C}(6)-\mathrm{C}(1)-\mathrm{C}(14)$ & $120.8(2)$ & $\mathrm{N}(3)-\mathrm{C}(14)-\mathrm{O}(2)$ & $118.50(19)$ \\
\hline $\mathrm{C}(2)-\mathrm{C}(1)-\mathrm{C}(14)$ & $120.6(2)$ & $\mathrm{N}(3)-\mathrm{C}(14)-\mathrm{C}(1)$ & $122.8(2)$ \\
\hline$C(3)-C(2)-C(1)$ & $120.2(3)$ & $\mathrm{O}(2)-\mathrm{C}(14)-\mathrm{C}(1)$ & $118.65(18)$ \\
\hline $\mathrm{C}(3)-\mathrm{C}(2)-\mathrm{H}(2)$ & 119.9 & $\mathrm{C}(16)-\mathrm{C}(15)-\mathrm{H}(15 \mathrm{~A})$ & 109.5 \\
\hline $\mathrm{C}(1)-\mathrm{C}(2)-\mathrm{H}(2)$ & 119.9 & $\mathrm{C}(16)-\mathrm{C}(15)-\mathrm{H}(15 \mathrm{~B})$ & 109.5 \\
\hline $\mathrm{C}(4)-\mathrm{C}(3)-\mathrm{C}(2)$ & $120.6(3)$ & $\mathrm{H}(15 \mathrm{~A})-\mathrm{C}(15)-\mathrm{H}(15 \mathrm{~B})$ & 109.5 \\
\hline $\mathrm{C}(4)-\mathrm{C}(3)-\mathrm{H}(3)$ & 119.7 & $\mathrm{C}(16)-\mathrm{C}(15)-\mathrm{H}(15 \mathrm{C})$ & 109.5 \\
\hline $\mathrm{C}(2)-\mathrm{C}(3)-\mathrm{H}(3)$ & 119.7 & $\mathrm{H}(15 \mathrm{~A})-\mathrm{C}(15)-\mathrm{H}(15 \mathrm{C})$ & 109.5 \\
\hline $\mathrm{C}(3)-\mathrm{C}(4)-\mathrm{C}(5)$ & $120.0(2)$ & $\mathrm{H}(15 \mathrm{~B})-\mathrm{C}(15)-\mathrm{H}(15 \mathrm{C})$ & 109.5 \\
\hline $\mathrm{C}(3)-\mathrm{C}(4)-\mathrm{H}(4)$ & 120.0 & $\mathrm{~N}(4)-\mathrm{C}(16)-\mathrm{C}(15)$ & $109.69(16)$ \\
\hline $\mathrm{C}(5)-\mathrm{C}(4)-\mathrm{H}(4)$ & 120.0 & $\mathrm{~N}(4)-\mathrm{C}(16)-\mathrm{C}(20)$ & $109.54(14)$ \\
\hline $\mathrm{C}(4)-\mathrm{C}(5)-\mathrm{C}(6)$ & $120.0(3)$ & $C(15)-C(16)-C(20)$ & $110.79(18)$ \\
\hline $\mathrm{C}(4)-\mathrm{C}(5)-\mathrm{H}(5)$ & 120.0 & $\mathrm{~N}(4)-\mathrm{C}(16)-\mathrm{C}(17)$ & $108.32(16)$ \\
\hline $\mathrm{C}(6)-\mathrm{C}(5)-\mathrm{H}(5)$ & 120.0 & $C(15)-C(16)-C(17)$ & $108.16(16)$ \\
\hline$C(1)-C(6)-C(5)$ & $120.6(2)$ & $C(20)-C(16)-C(17)$ & $110.28(17)$ \\
\hline $\mathrm{C}(1)-\mathrm{C}(6)-\mathrm{H}(6)$ & 119.7 & $C(18)-C(17)-C(16)$ & $114.15(16)$ \\
\hline $\mathrm{C}(5)-\mathrm{C}(6)-\mathrm{H}(6)$ & 119.7 & $\mathrm{C}(18)-\mathrm{C}(17)-\mathrm{H}(17 \mathrm{~A})$ & 108.7 \\
\hline $\mathrm{N}(1)-\mathrm{C}(7)-\mathrm{O}(1)$ & $121.5(2)$ & $\mathrm{C}(16)-\mathrm{C}(17)-\mathrm{H}(17 \mathrm{~A})$ & 108.7 \\
\hline $\mathrm{N}(1)-\mathrm{C}(7)-\mathrm{C}(8)$ & $120.2(2)$ & $\mathrm{C}(18)-\mathrm{C}(17)-\mathrm{H}(17 \mathrm{~B})$ & 108.7 \\
\hline $\mathrm{O}(1)-\mathrm{C}(7)-\mathrm{C}(8)$ & $118.27(15)$ & $\mathrm{C}(16)-\mathrm{C}(17)-\mathrm{H}(17 \mathrm{~B})$ & 108.7 \\
\hline $\mathrm{C}(13)-\mathrm{C}(8)-\mathrm{C}(9)$ & $118.8(2)$ & $\mathrm{H}(17 \mathrm{~A})-\mathrm{C}(17)-\mathrm{H}(17 \mathrm{~B})$ & 107.6 \\
\hline $\mathrm{C}(13)-\mathrm{C}(8)-\mathrm{C}(7)$ & $120.44(17)$ & $\mathrm{N}(2)-\mathrm{C}(18)-\mathrm{C}(17)$ & $115.6(2)$ \\
\hline $\mathrm{C}(9)-\mathrm{C}(8)-\mathrm{C}(7)$ & $120.7(2)$ & $\mathrm{N}(2)-\mathrm{C}(18)-\mathrm{C}(19)$ & $123.2(2)$ \\
\hline $\mathrm{C}(10)-\mathrm{C}(9)-\mathrm{C}(8)$ & $120.5(2)$ & $C(17)-C(18)-C(19)$ & $121.14(18)$ \\
\hline \multirow[t]{2}{*}{$\mathrm{C}(10)-\mathrm{C}(9)-\mathrm{H}(9)$} & 119.8 & $\mathrm{C}(18)-\mathrm{C}(19)-\mathrm{H}(19 \mathrm{~A})$ & 109.5 \\
\hline & & $\mathrm{C}(18)-\mathrm{C}(19)-\mathrm{H}(19 \mathrm{~B})$ & 109.5 \\
\hline
\end{tabular}




\begin{tabular}{|c|c|}
\hline $\mathrm{C}\left(30^{\prime}\right)-\mathrm{C}\left(31^{\prime}\right)-\mathrm{H}(31 \mathrm{C})$ & 109.5 \\
\hline $\mathrm{C}\left(32^{\prime}\right)-\mathrm{C}\left(31^{\prime}\right)-\mathrm{H}(31 \mathrm{C})$ & 109.5 \\
\hline $\mathrm{C}\left(30^{\prime}\right)-\mathrm{C}\left(31^{\prime}\right)-\mathrm{H}(31 \mathrm{D})$ & 109.5 \\
\hline $\mathrm{C}\left(32^{\prime}\right)-\mathrm{C}\left(31^{\prime}\right)-\mathrm{H}(31 \mathrm{D})$ & 109.5 \\
\hline $\mathrm{H}(31 \mathrm{C})-\mathrm{C}\left(31^{\prime}\right)-\mathrm{H}(31 \mathrm{D})$ & 108.0 \\
\hline $\mathrm{C}\left(33^{\prime}\right)-\mathrm{C}\left(32^{\prime}\right)-\mathrm{C}\left(31^{\prime}\right)$ & $110.5(4)$ \\
\hline $\mathrm{C}\left(33^{\prime}\right)-\mathrm{C}\left(32^{\prime}\right)-\mathrm{H}(32 \mathrm{C})$ & 109.5 \\
\hline $\mathrm{C}\left(31^{\prime}\right)-\mathrm{C}\left(32^{\prime}\right)-\mathrm{H}(32 \mathrm{C})$ & 109.5 \\
\hline $\mathrm{C}\left(33^{\prime}\right)-\mathrm{C}\left(32^{\prime}\right)-\mathrm{H}(32 \mathrm{D})$ & 109.5 \\
\hline $\mathrm{C}\left(31^{\prime}\right)-\mathrm{C}\left(32^{\prime}\right)-\mathrm{H}(32 \mathrm{D})$ & 109.5 \\
\hline $\mathrm{H}(32 \mathrm{C})-\mathrm{C}\left(32^{\prime}\right)-\mathrm{H}(32 \mathrm{D})$ & 108.1 \\
\hline $\mathrm{C}\left(32^{\prime}\right)-\mathrm{C}\left(33^{\prime}\right)-\mathrm{C}\left(34^{\prime}\right)$ & $111.5(4)$ \\
\hline $\mathrm{C}\left(32^{\prime}\right)-\mathrm{C}\left(33^{\prime}\right)-\mathrm{H}(33 \mathrm{C})$ & 109.3 \\
\hline $\mathrm{C}\left(34^{\prime}\right)-\mathrm{C}\left(33^{\prime}\right)-\mathrm{H}(33 \mathrm{C})$ & 109.3 \\
\hline $\mathrm{C}\left(32^{\prime}\right)-\mathrm{C}\left(33^{\prime}\right)-\mathrm{H}(33 \mathrm{D})$ & 109.3 \\
\hline $\mathrm{C}\left(34^{\prime}\right)-\mathrm{C}\left(33^{\prime}\right)-\mathrm{H}(33 \mathrm{D})$ & 109.3 \\
\hline $\mathrm{H}(33 \mathrm{C})-\mathrm{C}\left(33^{\prime}\right)-\mathrm{H}(33 \mathrm{D})$ & 108.0 \\
\hline $\mathrm{C}\left(33^{\prime}\right)-\mathrm{C}\left(34^{\prime}\right)-\mathrm{C}\left(35^{\prime}\right)$ & $111.0(4)$ \\
\hline $\mathrm{C}\left(33^{\prime}\right)-\mathrm{C}\left(34^{\prime}\right)-\mathrm{H}(34 \mathrm{C})$ & 109.4 \\
\hline $\mathrm{C}\left(35^{\prime}\right)-\mathrm{C}\left(34^{\prime}\right)-\mathrm{H}(34 \mathrm{C})$ & 109.4 \\
\hline $\mathrm{C}\left(33^{\prime}\right)-\mathrm{C}\left(34^{\prime}\right)-\mathrm{H}(34 \mathrm{D})$ & 109.4 \\
\hline $\mathrm{C}\left(35^{\prime}\right)-\mathrm{C}\left(34^{\prime}\right)-\mathrm{H}(34 \mathrm{D})$ & 109.4 \\
\hline $\mathrm{H}(34 \mathrm{C})-\mathrm{C}\left(34^{\prime}\right)-\mathrm{H}(34 \mathrm{D})$ & 108.0 \\
\hline $\mathrm{C}\left(34^{\prime}\right)-\mathrm{C}\left(35^{\prime}\right)-\mathrm{C}\left(30^{\prime}\right)$ & $111.3(4)$ \\
\hline $\mathrm{C}\left(34^{\prime}\right)-\mathrm{C}\left(35^{\prime}\right)-\mathrm{H}(35 \mathrm{C})$ & 109.4 \\
\hline $\mathrm{C}\left(30^{\prime}\right)-\mathrm{C}\left(35^{\prime}\right)-\mathrm{H}(35 \mathrm{C})$ & 109.4 \\
\hline $\mathrm{C}\left(34^{\prime}\right)-\mathrm{C}\left(35^{\prime}\right)-\mathrm{H}(35 \mathrm{D})$ & 109.4 \\
\hline $\mathrm{C}\left(30^{\prime}\right)-\mathrm{C}\left(35^{\prime}\right)-\mathrm{H}(35 \mathrm{D})$ & 109.4 \\
\hline $\mathrm{H}(35 \mathrm{C})-\mathrm{C}\left(35^{\prime}\right)-\mathrm{H}(35 \mathrm{D})$ & 108.0 \\
\hline
\end{tabular}

Symmetry transformations used to generate equivalent atoms: 
$\mathrm{S} 15$

Table 4. Anisotropic displacement parameters $\left(\mathrm{pm}^{2} \times 10^{-1}\right)$ for $\mathbf{3 b}$. The anisotropic displacement factor exponent takes the form: $-2 \pi^{2}\left[h^{2} a^{* 2} U^{11}+\ldots+2 h k a^{*} b^{*} U^{12}\right]$

\begin{tabular}{|c|c|c|c|c|c|c|}
\hline & $\mathrm{U}^{11}$ & $\mathrm{U}^{22}$ & $\mathrm{U}^{33}$ & $\mathrm{U}^{23}$ & $\mathrm{U}^{13}$ & $\mathrm{U}^{12}$ \\
\hline $\mathrm{C}(1)$ & $128(2)$ & $29(1)$ & $34(1)$ & $-9(1)$ & $-2(1)$ & $-2(1)$ \\
\hline$C(2)$ & $148(2)$ & $38(1)$ & $33(1)$ & $-5(1)$ & $-9(1)$ & $-8(1)$ \\
\hline$C(3)$ & $178(3)$ & $41(1)$ & $35(1)$ & $-4(1)$ & $-6(1)$ & $-13(2)$ \\
\hline$C(4)$ & $182(3)$ & $41(1)$ & $41(1)$ & $-5(1)$ & $15(2)$ & $-17(2)$ \\
\hline$C(5)$ & $144(2)$ & $50(1)$ & $54(1)$ & $-4(1)$ & $14(1)$ & $-9(1)$ \\
\hline$C(6)$ & $133(2)$ & $46(1)$ & $42(1)$ & $-3(1)$ & $0(1)$ & $-4(1)$ \\
\hline$C(7)$ & $104(2)$ & $34(1)$ & $39(1)$ & $6(1)$ & $-12(1)$ & $-11(1)$ \\
\hline $\mathrm{C}(8)$ & $112(2)$ & $35(1)$ & $33(1)$ & $-1(1)$ & $-6(1)$ & $1(1)$ \\
\hline $\mathrm{C}(9)$ & $126(2)$ & $49(1)$ & $35(1)$ & $4(1)$ & $2(1)$ & $20(1)$ \\
\hline$C(10)$ & $155(2)$ & $66(1)$ & $41(1)$ & $9(1)$ & $7(1)$ & $43(2)$ \\
\hline $\mathrm{C}(11)$ & $150(2)$ & $67(2)$ & $54(1)$ & $-2(1)$ & $11(1)$ & $48(2)$ \\
\hline $\mathrm{C}(12)$ & $140(2)$ & $60(1)$ & $41(1)$ & $-7(1)$ & $11(1)$ & $21(1)$ \\
\hline $\mathrm{C}(13)$ & $126(2)$ & $44(1)$ & $34(1)$ & $0(1)$ & $-5(1)$ & $0(1)$ \\
\hline $\mathrm{C}(14)$ & $113(2)$ & $30(1)$ & $34(1)$ & $-7(1)$ & $-10(1)$ & $-4(1)$ \\
\hline$C(15)$ & $118(2)$ & $43(1)$ & $52(1)$ & 1(1) & $-19(1)$ & $6(1)$ \\
\hline$C(16)$ & $97(1)$ & $35(1)$ & $40(1)$ & $6(1)$ & $-16(1)$ & $-5(1)$ \\
\hline $\mathrm{C}(17)$ & $105(2)$ & $42(1)$ & $55(1)$ & $21(1)$ & $-24(1)$ & $-9(1)$ \\
\hline$C(18)$ & $100(2)$ & $47(1)$ & $51(1)$ & $23(1)$ & $-22(1)$ & $-19(1)$ \\
\hline C(19) & $107(2)$ & $87(2)$ & $52(1)$ & $28(1)$ & $-17(1)$ & $-9(2)$ \\
\hline$C(20)$ & $97(2)$ & $45(1)$ & $37(1)$ & $1(1)$ & $-9(1)$ & $-7(1)$ \\
\hline $\mathrm{N}(1)$ & $100(1)$ & $47(1)$ & $45(1)$ & $15(1)$ & $-19(1)$ & $-8(1)$ \\
\hline $\mathrm{N}(2)$ & $95(1)$ & $44(1)$ & $48(1)$ & $17(1)$ & $-17(1)$ & $-12(1)$ \\
\hline $\mathrm{N}(3)$ & $109(1)$ & $35(1)$ & $35(1)$ & $-1(1)$ & $-7(1)$ & $-7(1)$ \\
\hline $\mathrm{N}(4)$ & 101(1) & $33(1)$ & $37(1)$ & $3(1)$ & $-13(1)$ & $-9(1)$ \\
\hline $\mathrm{O}(1)$ & $102(1)$ & $36(1)$ & $39(1)$ & $10(1)$ & $-9(1)$ & $-3(1)$ \\
\hline $\mathrm{O}(2)$ & $115(1)$ & $40(1)$ & $32(1)$ & $-3(1)$ & $-13(1)$ & $2(1)$ \\
\hline $\operatorname{Si}(1)$ & $104(1)$ & $28(1)$ & $41(1)$ & $6(1)$ & $-20(1)$ & $-7(1)$ \\
\hline $\mathrm{C}(30)$ & $75(3)$ & $29(2)$ & $25(2)$ & $-1(1)$ & $5(2)$ & $1(2)$ \\
\hline $\mathrm{C}(31)$ & $110(4)$ & $37(2)$ & $62(3)$ & $-13(2)$ & $-27(3)$ & $7(2)$ \\
\hline $\mathrm{C}(32)$ & $98(3)$ & $45(2)$ & $65(2)$ & $-10(2)$ & $-23(2)$ & $5(2)$ \\
\hline $\mathrm{C}(33)$ & $96(3)$ & $46(2)$ & $43(2)$ & $-15(2)$ & $0(2)$ & $-9(2)$ \\
\hline $\mathrm{C}(34)$ & $90(3)$ & $34(2)$ & $72(3)$ & $-11(2)$ & $-6(2)$ & $-2(2)$ \\
\hline$C(35)$ & $83(3)$ & $43(2)$ & $62(2)$ & $-15(2)$ & $1(2)$ & $-3(2)$ \\
\hline $\mathrm{C}\left(30^{\prime}\right)$ & $64(3)$ & $29(2)$ & $30(2)$ & $-5(2)$ & $3(2)$ & $-1(2)$ \\
\hline$C\left(31^{\prime}\right)$ & $104(3)$ & $40(2)$ & $39(2)$ & $0(2)$ & $8(2)$ & $-18(2)$ \\
\hline
\end{tabular}




\begin{tabular}{lllllll} 
& \multicolumn{5}{c}{$\mathrm{S} 16$} \\
$\mathrm{C}\left(32^{\prime}\right)$ & $105(4)$ & $45(2)$ & $50(3)$ & $-4(2)$ & $9(3)$ & $-27(3)$ \\
$\mathrm{C}\left(33^{\prime}\right)$ & $80(4)$ & $53(3)$ & $65(3)$ & $-15(3)$ & $2(3)$ & $-8(3)$ \\
$\mathrm{C}\left(34^{\prime}\right)$ & $90(3)$ & $49(3)$ & $46(2)$ & $-5(2)$ & $-10(2)$ & $-8(2)$ \\
$\mathrm{C}\left(35^{\prime}\right)$ & $77(3)$ & $43(2)$ & $41(2)$ & $-1(2)$ & $-7(2)$ & $4(2)$ \\
\hline
\end{tabular}

